\title{
A Predictive Heat Generation Model in Orthogonal Cutting Visco-plastic Material
}

\author{
Yuliu Zheng, John W. Sutherland and Walter W. Olson \\ Department of Mechanical Engineering \& Engineering Mechanics \\ Michigan Technological University \\ Houghton, MI 49931, USA
}

\section{Abstract}

The mechanics of the orthogonal cutting process are examined. Under an assumed streamline and shear plane curvilinear system, the deformation rate tensor in the primary zone is obtained analytically. The secondary zone is treated as a plastic boundary layer, in which the flow field of velocity and deformation rate is also obtained. The effect of the temperature distribution on the dissipated work done in machining is included. The shear angle, cutting forces, and the intensity and shape of the heat sources in orthogonal cutting are predicted by the total work minimization principle and compared with predictions from the literature.

\subsection{Introduction}

Machining, or material removal, operations are widely employed in industry for the production of a variety of engineered products. Traditional machining operations such as turning, milling, and broaching remove/cut material through a shearing process. The performance of these cutting operations is often characterized by such measures as machined surface finish, cutting force, tool life, and the part dimensions produced by the process. All of these measures are affected to some extent by the heat generated during the cutting process. Additionally, the recent focus of manufacturers on reducing/ameliorating their waste streams has generated significant interest in the relationship between cutting fluids and heat transfer in machining. As a step toward describing this relationship, a fundamental understanding of the heat generation mechanism in cutting is desired.

It is known that the heat generated in machining results from the plastic work done in the primary and secondary zones, viscous dissipation if the work material is visco-plastic, and the frictional work done along the tool/chip and tool/workpiece interfaces. To quantify these components, attention must be focused on the mechanics of machining. A review of the technical literature reveals that generally, orthogonal cutting, plane strain, and steady-state conditions have been assumed for the development of machining theory. Due to the very large strains, high strain rates, high temperatures, and complex stress states occurring in machining processes, even with the above simplications, the mechanics of machining are not well understood. The orthogonal cutting model proposed by Merchant [1945] has often been used as a basis for analyzing the heat sources in machining. In this model, there is a discontinuity in the velocity of chip when it undergoes deformation across the shear plane. For the model, the uniform heat source must lie in the shear plane since all shear deformation occurs in that plane (See Figure 1).

Boothroyd [1963] studied the effect of two kinds of distributed heat sources in the secondary zone on cutting temperatures. The first heat source considered was uniformly distributed over a rectangular area, while the second heat source had a triangular area with a maximum rate of heat generation at the tool tip decreasing linearly along the rake face to zero at 
the edge of the secondary zone. Moreover, he assumed that the heat generated was due to plastic deformation in the chip and that no heat was generated due to friction at the tool/chip interface. He found that the rectangular-shaped heat source gave better agreement with his experimental results and that Rapier's analytical method tended to overestimate the temperature at the tool/chip interface [Rapier, 1954; Boothroyd, 1963].

The finite element method has also been employed to characterize heat sources. Tay, et al. [1974, 1976], calculated temperature distributions in the chip, tool and workpiece using the flow fields obtained from a printed grid on the workpiece. This information was then combined with measured cutting forces to obtain the intensity and dimension of heat sources. A model of the machining process was developed to describe the velocity, strain and strain rate distributions within the primary and secondary zones as a function of basic cutting parameters such as rake angle, shear plane angle and feed rate. A hyperbolic streamline based on the cutting conditions was proposed to describe the flow of chip material, from which the distributions of strain and strain rate were obtained and found similar to the experimental results of Stevenson and Oxley [Tay, et al, 1976; Stevenson and Oxley, 1979-1980].

A disadvantage of the model by Tay et al. is that unidirectional simple shear is assumed to occur in the primary zone, while in fact a complex stress state exists in the zone. Another drawback of the work is that the strain and strain rate are defined based on small deformation gradient theory, but the strains in cutting are very large. Additionally, the assumption that all the streamlines are identical implies that all the stress states along or parallel to the shear plane are the same. In general, this is not the case. Finally, the heat source model in the secondary zone was mainly based on experimental data.

The efforts to be described herein assume a streamline modified from Tay's, which allows the curvature of the streamlines to vary along the direction parallel to the shear plane. Under the streamline curvilinear coordinate system and body-oriented orthogonal system, the deformation rate tensors in the primary zone and the secondary zone are obtained using Mathematica [Wolfram,1993]. A constitutive equation for work materials of visco-plastic type is assumed. This equation is capable of considering large deformation gradients because the deformation rate tensor is used instead of strain and strain rate. The temperature distribution, shear plane angle, the intensity and the shape of the heat sources are estimated by minimizing the total work in the cutting process.

\subsection{Deformation and Thermal Analyses}

\subsection{The Primary Zone}

Geometry and coordinate system Consider the orthogonal cutting and plane strain problem shown in Figure 1. The curvilinear coordinate system is set on the assumed streamline and in the direction parallel to the so-called shear plane. The covariant base vectors are denoted by $\left(\boldsymbol{g}_{1}, \boldsymbol{g}_{2}\right)$.

The relationship between the coordinates $\left(q^{1}, q^{2}\right)$ and $\left(x^{1}, x^{2}\right)$ satisfy the following equations: 


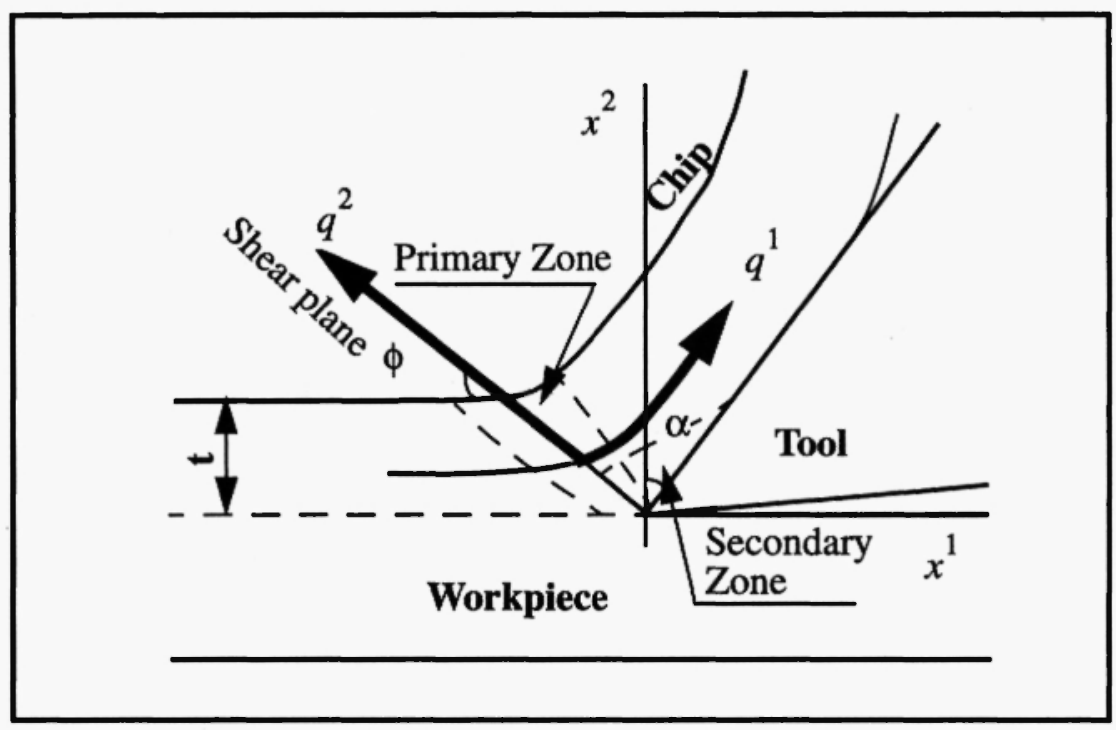

Figure 1. Orthogonal cutting geometry and coordinate system

$$
\begin{aligned}
& \left(x^{2}-q^{2} t\right)^{2} \tan \alpha-\left(x^{2}-q^{2} t\right)\left(x^{1}+q^{2} t \cot \phi\right)=a t^{2}\left(1+\eta q^{2}\right) \\
& t q^{1}=x^{2}+\tan \phi x^{1}
\end{aligned}
$$

where $t$ is the uncut chip thickness, a and $\eta$ are constants related to the cut material and cutting conditions, $\alpha$ and $\phi$ are the rake angle and the so-called shear plane angle, and the coordinates $\left(x^{1}, x^{2}\right)$ are in the Cartesian coordinate system which is a reference frame with base vectors $\left(i_{1}, i_{2}\right)$. The coordinates $\left(x^{1}, x^{2}\right)$ can be expressed in terms of $\left(q^{1}, q^{2}\right)$ as follows,

$$
\begin{aligned}
& x^{1}=t\left[\frac{(\beta-1) q^{1}-\sqrt{\Delta}}{\beta \tan \phi}-q^{2} \cot \phi\right] \\
& x^{2}=t\left[\frac{q^{1}+\sqrt{\Delta}}{\beta}+q^{2}\right]
\end{aligned}
$$

where $\beta=2(\tan \alpha \tan \phi+1)$, and $\Delta=\left(q^{1}\right)^{2}+2 a \beta \tan \phi\left(1+\eta q^{2} \csc \phi\right)$.

Determining the Velocity, Deformation Rate Tensor, and Dissipated Work For steady state conditions, the continuity equation is,

$$
\nabla \cdot(\rho v)=0
$$

where $\rho$ if the density of work material, and $\mathbf{v}$ the velocity vector[Malvern, 1969].

In the curvilinear coordinate system, due to the assumption that $q^{2}=$ constant is a family of streamlines, the contravariant component of the velocity vector in $g_{2}$ direction will vanish. From Eq.(3), we can have, 


$$
v^{\langle 1\rangle}=\sqrt{\frac{g_{11}}{g}} \sin \phi V, \rho_{o} / \rho
$$

where $v^{\langle 1\rangle}$ is the physical component of velocity vector in $g_{1}$ direction, $g_{11}$ is one of the covariant components of tensor $\tilde{g}$, and $g$ the determinant of tensor $\tilde{g}$, while $\phi$ and $V_{o}$ are the shear angle and cutting speed. $\rho_{o}$ and $\rho$ are the initial and instantaneous density of work material respectively.

The limits of the velocity, when $q^{1}$ goes to infinity, are given by,

$$
\lim _{q^{1} \rightarrow-\infty} v^{\langle 1\rangle}=V_{o}, \quad \quad \lim _{q^{1} \rightarrow \infty} v^{\langle 1\rangle}=\frac{\rho_{o} V_{o} \sin \phi}{\rho_{c} \cos (\alpha-\phi)}=V_{c}
$$

where $V_{c}$, and $\rho_{c}$ are the velocity and final density of the chip. If $\rho_{c}=\rho_{o}$, i.e., the density of work material is constant, Eq.(5) is consistent with the velocity diagram in the classical shear plane model.

The deformation rate tensor, $\tilde{D}$, is calculated from,

$$
D_{i j}=\frac{1}{2}\left(v_{i, j}+v_{j, i}\right)
$$

where $D_{i j}$ is the covariant component of the tensor $\tilde{D}$, and $v_{i, j}$ is the covariant derivative of covariant component $v_{i}$ of velocity with respect to coordinate $q_{j}$.

If $\rho$ is constant, then

$$
\nabla \cdot v=\operatorname{tr}(\vec{D})=0
$$

therefore, the second invariant of tensor $\tilde{D}$ is

$$
I I_{D}=\frac{1}{2}\left(\tilde{D}: \tilde{D}-t r^{2} \tilde{D}\right)=\frac{1}{2} D^{i j} D_{i j}
$$

where $D^{i j}$ is the contravariant component of tensor $\tilde{D}$. Using Mathematica, the finite Eulerian strain and strain rate tensors, not used in this paper, can be analytically obtained also.

The constitutive equation used is in the following form,

$$
\bar{T}=\left(\mu_{o}+\frac{\tau_{o}}{\sqrt{I_{D}}}\right) \bar{D}
$$

where $\tilde{T}$ is the deviatric Cauchy stress tensor, both $\mu_{o}$ and $\tau_{o}$, which may vary with temperature, are the material functions, which depend on temperature, for visco-plastic material. The dissipated work, which finally converts into heat, is then given by

$$
\Psi_{p}=2 \mu_{o} I_{D}+2 \tau_{o} \sqrt{I_{D}} \text {. }
$$


Determining the Shape of the Primary Zone It is known that the deformation and heat generated mainly concentrate in a very narrow zone. Supposing that the 2 nd invariant of deformation rate tensor, $I I_{D}$, is regarded as the probabilistic distribution of a certain random variable, then the mean of this variable, $\bar{q}^{1}$, can identify the position of so-called shear plane, and a multiple of the variance, $\delta_{v}$, can be assumed as the thickness of primary zone,

$$
\bar{q}=\frac{\int q^{1} I I_{D} d q^{1}}{\int I_{D} d q^{1}}, \quad \delta_{v}=\left[\frac{\int\left(q^{1}-\overline{q^{1}}\right)^{2} I_{D^{2}} q^{1}}{\int I_{D} d q^{1}}\right]^{1 / 2} .
$$

Thermal Analysis in the Primary Zone From Booythroyd's experimental results [Boothroyd, 1963] and Tay et al's numerical results by finite element method [Tay, et al, 1976], it is safe to make an assumption that, in the primary shear zone $\left[\bar{q}^{1}-4 \delta_{v}, \bar{q}+4 \delta_{v}\right]$, the temperature, $\theta$, takes on the following distribution,

$$
\theta=\theta_{o}+\eta_{\theta}\left[\eta_{o}+\eta_{1} q^{1}+\left(q^{1}\right)^{2}\right]
$$

where $\theta_{o}$ is the initial temperature of the chip, $\eta_{o}$ and $\eta_{1}$ may vary in the direction parallel to shear plane, and can be determined by the following assumed conditions,

$$
\left.\theta\right|_{q^{1}=\vec{q}-4 \delta_{v}}=\theta_{o},\left.\quad \frac{\partial \theta}{\partial q^{1}}\right|_{q^{1}=\underline{a}+4 \delta_{v}}=0
$$

And $\eta_{\theta}$ is a constant in the primary shear zone, which can be obtained by applying the energy equation,

$$
\rho C_{p} \nu \cdot \nabla \theta=\nabla \cdot(k \nabla \theta)+\psi_{p}
$$

where $C_{p}$ and $k$ are the specific heat capacity and thermal conductivity. Because the dissipated work is dependent upon temperature, an iterative method is used to calculate $\eta_{\theta}$. Theoretically, Eq.[13] should be satisfied at the every points in the primary zone, but $\eta_{\theta}$ is only the average value taken from several points on the shear plane. If the assumed streamline and the constitutive equation are known, the dissipated work in the primary zone, which depends on both the deformation rate and temperature, can be obtained.

\subsection{The Secondary Zone}

Due to the complexity of the deformation and thermal processes in the secondary zone, no comprehensive theoretical analysis is available in the literature. Jackson and Wright, Kim and Marinov applied Oldroyd's solution for the plastic boundary layer near a flat knife in describing the flow behavior of cut material near the chip/tool interface [Oldroyd,1947; Jackson and Wright, 1982; Kim and Marinov, 1995]. The tool-face pressure gradient, average boundary-layer thickness, shear strain rate and shear flow strength were obtained under limiting assumptions. The assertion that the inertia effect can be completely ignored is very reasonable because of very low Reynold number found in machining. The assumption that the velocity at the upper side of the 
boundary layer is constant is also acceptable (See Figure 3). But Oldroyd's similarity solution may not exist if the thermal behavior is considered. The fact that the thickness of the secondary zone decreases with distance from the tool tip indicates that Oldroyd's solution may not directly be applied in the secondary zone. In this work, the region between the last streamline and the chip/tool interface is treated as the plastic boundary layer. The thickness of the boundary layer is simply determined as the distance from a point on the streamline to the chip/tool interface. A schematic is shown in Figure 2.

Determine the Thickness of Boundary Layer A schematic of the secondary zone or assumed plastic boundary layer is shown in Figure 2. In the reference frame, the coordinates $\left(x_{A}{ }^{1}, x_{A}{ }^{2}\right)$ of point $\mathrm{A}$ is given by Eq.(2) in terms of $\left(q^{1}, q^{2 *}\right)$. The line $\mathrm{AB}$ is perpendicular to $\mathrm{QB}$ and $\mathrm{B}$ is the intersection whose coordinates $\left(x_{B}{ }^{1}, x_{B}{ }^{2}\right)$ are readily calculated in terms of $\left(q^{1}, q^{2 *}\right)$, therefore, we have

$$
\delta=\sqrt{\left|r_{A}-r_{B}\right|}=\delta\left(q^{i}, q^{i+i}\right)
$$

where $\boldsymbol{r}_{A}$, and $\boldsymbol{r}_{B}$ are the position vectors of A, B respectively.

In addition, we have

$$
x=x_{B}{ }^{1}\left(q^{1}, q^{2 *}\right) / \sin \alpha
$$

Eq.(15) can be rewritten as $q^{1}$ in terms of $x$, then substituting it into Eq.(14), the thickness of boundary layer, $\delta$, can be expressed in terms of $\mathbf{x}$.

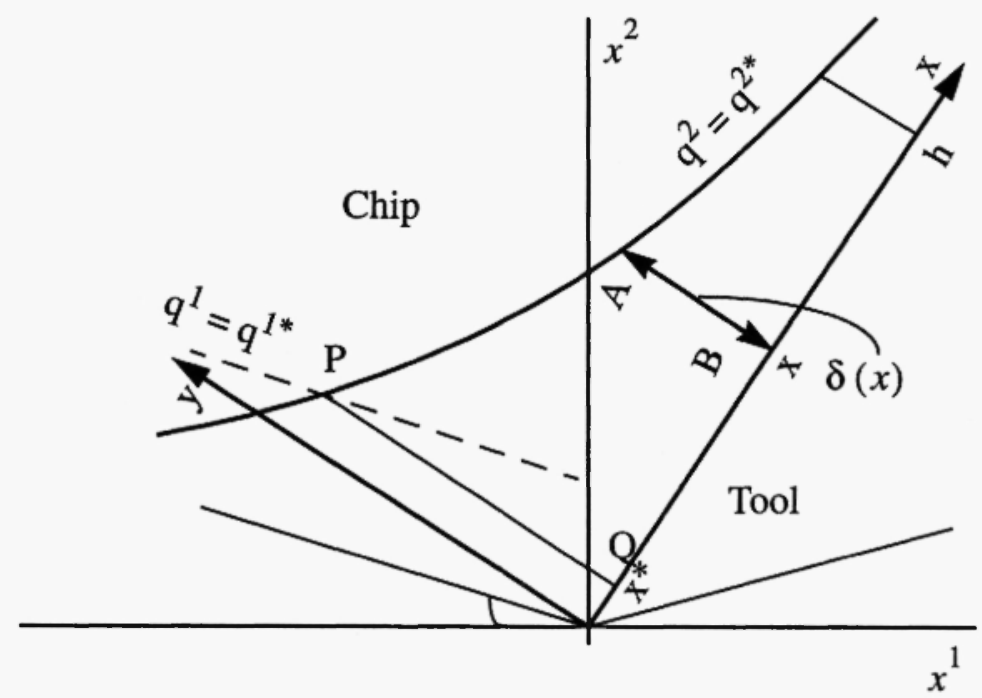

Figure 2 A schematic of the secondary zone as boundary layer: $q^{1 *}$ indicates the end of the primary zone, and $q^{2 *}$ indicates the last streamline in the primary zone, while $x^{*}$ is the starting point of the secondary zone and $\delta(x)$ the thickness of the secondary zone or boundary layer at $x$. 
Velocity Field and Deformation Rate Tensor in the Secondary Zone The velocity is assumed to be of the form,

$$
\begin{aligned}
& v_{x}=V_{c}+\left(V_{w}-V_{c}\right)\left(1-\frac{y}{\delta}\right)^{2} \\
& v_{y}=-\frac{\partial}{\partial x}\left[\left(V_{w}-V_{s}\right)\left(y-\frac{y^{2}}{\delta}+\frac{y^{3}}{3 \delta^{2}}\right)\right]
\end{aligned}
$$

where $v_{x}$ and $v_{y}$ are components of velocity vector and $V_{c}$ is the chip velocity. $V_{w}$ is the velocity distribution along the chip/tool interface and may be characterized as follows,

$$
V_{w}=V_{i}\left[\frac{1}{\bar{z}}+\frac{1}{\pi} \tan : \omega\left(\frac{\ddot{\bar{h}}}{\bar{h}}-b\right)\right] \text {. }
$$

If the parameter $b>0.5$, the velocity $V_{w}$ tends to zero, or seizes, on the first half of the tool-chip contact. If the parameter $\omega$ is large enough, $V_{w}$ goes to the chip velocity at the end of contact length. In addition, Eqs.(18-19) satisfy the continuity equation,Eq.(3), and the following boundary conditions,

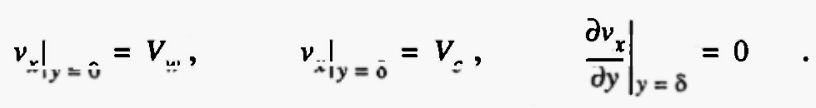

These boundary conditions make the momentum equations in Oldroyd's form non-singular at the upper side of plastic boundary layer. One boundary condition, namely $\left.v_{y}\right|_{v=\delta}=0$, remains unsatisfied. The same problem is also met in Prandtl's boundary-layer theory [Oldroyd,1947]. In fact, the numerical results indicate that the velocity component, $v_{y !_{y}}=\delta$, is about $2 \%$ of the final chip velocity.

The deformation rate tensor and its 2 nd invariant are then

$$
\begin{aligned}
& d_{x x}=\frac{\partial v_{*}}{\partial x}, \quad d_{x y}=\frac{1}{2}\left(\frac{\partial v_{x}}{\partial y}+\frac{\partial v_{y}}{\partial x}\right), \quad d_{y y}=\frac{\partial v_{y}}{\partial y} \\
& I_{d}=\frac{1}{2} d_{i j} d_{i j}, \quad\left(d_{i i}=0\right) .
\end{aligned}
$$

The dissipated work done in the secondary zone is given by

$$
\Psi_{s}=2 \mu_{o} I I_{d}+2 \tau_{o} \sqrt{I I_{d}}
$$

The friction work generated at the chip/tool interface is

$$
\Psi_{f}=\left.V_{w} \boldsymbol{\tau}_{x y}\right|_{y=0}
$$

where, from Eq.(9), the shear stress at the interface is given by

$$
\left.\tau_{x y}\right|_{y=0}=\left.\left(\mu_{0}+\frac{\tau_{o}}{\sqrt{I_{d}}}\right) d_{x y}\right|_{y=0}
$$

Thermal Analysis in the Secondary Zone There is a large temperature gradient in the secondary zone and the method typically used to calculate the temperature in the primary zone gives poor results in the secondary zone. In order to obtain the temperature distribution in this zone, it is 
necessary to use a numerical method together with some information on the heat source or completely apply a thermal-mechanical coupled method [Lin and Pan, 1993; Marusich and Ortiz, 1995]. As a semi-analytical predictive model for heat generation, a simple method is introduced to estimate the temperature distribution in the secondary zone.

Based on previous knowledge, several assumptions about the temperature distributions in the secondary zone are made:

(1). At $x=x^{*}$, the temperature, $\theta^{*}$, at both the lower and upper sides of boundary layer are same and can be determined by Eq.(15), i.e., $\theta^{*}=\theta\left(q^{1 *}, q^{2 *}\right)$.

(2). At $x=h$, the end of contact length, the temperature, $\theta_{1}$, at both the lower and upper sides of boundary layer are also same and equal to the final average temperature in the chip.

(3). At $x=h$, the temperature gradient in $\mathrm{x}$ direction tends to zero, i.e., $\left.\frac{\partial \theta}{\partial x}\right|_{x=h} \approx 0$.

(4). At $y=\delta(x)$, the upper side of boundary layer, the temperature gradient is also zero, namely $\left.\frac{\partial \theta}{\partial y}\right|_{y=\delta}=0$.

(5). At $y=0$, the chip/tool interface or lower side of boundary layer, the maximum temperature is approximately appeared at half the contact length, $x=h / 2$.

(6). The temperature rise, $\Delta \theta=\theta_{1}-\theta^{*}$, is because the dissipated work, $W_{3}$, done in the secondary zone and the friction work, $W_{f}$, generated at the tool/chip interface are completely converted into heat, therefore

$$
\Delta \theta=\frac{w_{s}+w_{J}}{\rho C_{p} w t V_{o}}
$$

where $w$ is the width of cutting, and $h$ the contact length, and

$$
W_{s}=w \int_{x^{*}}^{h} \int_{0}^{\delta_{s}} \Psi_{s} d y d x, \quad W_{f}=w \int_{0}^{h} \Psi_{f} d x .
$$

Under the above assumptions, the temperature distributions at the lower and upper sides of boundary layer as well as inside the secondary zone are further simplified as follows:

$$
\begin{aligned}
& \theta_{u}=\left.\theta\right|_{y=\delta}=\theta^{*}+\left(\theta_{1}-\theta^{*}\right)\left(2 \xi-\xi^{2}\right) \\
& \theta_{l}=\left.\theta\right|_{y=n}=\theta^{*}+\left(\theta_{1}-\theta^{*}\right)\left(1-e^{-\frac{\pi}{2} \xi} \cos 2 \pi \xi\right)\left(1-e^{-\frac{\pi}{2}}\right) \\
& \theta=\theta_{u}+\left(\theta_{l}-\theta_{u}\right)\left(1-\frac{y}{\delta}\right)^{2}
\end{aligned}
$$

where the parameter $\xi=\left(x-x^{*}\right) / h$.

It should be noted that in Eq. (23), $W_{s}$ and $W_{f}$ are both dependent upon temperature in the secondary zone and at the chip/tool interface, so in order to calculate $\theta_{1}$, another iterative algorithm is needed. 


\subsection{The Total Work Done and its Minimization}

The overall work per unit width of cutting, $W$, done in machining is the sum of that done in the primary zone, secondary zone as well as at the chip/tool interface, that is,

$$
W=\int \Psi_{p} J d q^{1} d q^{2}+\int \Psi_{s} d x d y+\int \Psi_{f} d x
$$

where $J$ is the Jacobian determinant and given by $J=\frac{\partial\left(x^{\prime} x^{2}\right)}{\partial\left(q^{1}, q^{2}\right)}$. Noting that, in the $q$ coordinate system, the integral limits in $q^{2}$ direction is not easy to accurately assigned, there is an error to integrate the first term in above equation; and that the integral limits in $q^{1}$ direction is the thickness of the primary zone determined from Eq. (14); and that the integral regions of the first two terms in Eq.(25) may overlap a little bit or cannot cover the whole primary and secondary zone, which case occurred will depend upon the tool geometry as well as cutting conditions.

Obviously, $W$ is a function of five variables: $\phi, a, \eta, q^{2 *}$ and $h$, but these variables are not independent.Tay et al. related $a$ to $\phi$ in the following way,

$$
a=\frac{1}{16 C^{2} \sin ^{4} \phi(\tan \alpha+\cot \phi)}
$$

where $\mathrm{C}$ is a constant determined by experiment, In this paper, $\mathrm{C}$ will be obtained by overall work minimization. In addition, Hastings et al. proposed that the contact length, h, can approximated as

$$
h=\frac{t \sin (\phi+\lambda-\alpha)}{\cos \lambda \sin \alpha}
$$

where $\lambda$ is the mean friction angle over the chip/tool interface. Under seizure conditions, $\lambda$ is chosen such that $\tan \lambda=0.8$ in this work.

The work, $\mathrm{W}$, is then rewritten as: $W=W\left(\phi, C, \eta, q^{2 *}\right)$. These four variables may be regarded as independent. It should be noted that $\eta_{\theta}$ in Eq.(12) and $\theta_{1}$ in Eq.(24) do not explicitly appeare in W, because these two parameters can be determined by using an iteration method if $\phi$, $\mathrm{C}, \eta$ and $q^{2 *}$ are known. Moreover, the classical machining theory and experimental results are helpful to determine the possible range of above parameters and to verify the predicted results. When $\mathrm{W}$ is minimized, the corresponding outputs are then used to calculate cutting forces, power consumption, and other model outputs.

\subsection{Numerical Results and Discussion}

The work material used for the following numerical calculation is plain carbon steel. A set of data for its viscosity coefficient and low strain-rate shear strength has been obtained from Hopkinson-bar tests by Cambell and Ferguson. Accordingly, $\mu_{o}$ and $\tau_{o}$ vary with temperature as follows [Kim and Marinov, 1995], 


$$
\tau_{0}=240(293 / \theta)^{085}(\mathrm{MPa}), \quad \quad \mu_{s}=2.1(293 / \theta)^{0.17}(\mathrm{KPa} \mathrm{s}) .
$$

The thermal properties $C_{p}$ and $k$ for plain carbon steel are $486.0 \mathrm{~J} / \mathrm{Kg} \cdot \mathrm{K}$ and 54.17

$W / m \cdot K$ respectively, which are from the reference [Incropera and DeWitt, 1979] and the density is $7854 \mathrm{Kg} / \mathrm{m}^{3}$. The cutting conditions are listed in the following: $\alpha=5^{\circ}$, $V_{o}=1.67 \mathrm{~m} / \mathrm{s}, t=0.25 \mathrm{~mm}$, and the ambient temperature, $\theta_{o}$ in Eq.(14), is $22^{\circ} \mathrm{C}$. The parameters obtained by the total work minimization are given in Table 1 The velocity distribution

TABLE 1. Predicted Model Parameters

\begin{tabular}{|c|c|c|}
\hline$\phi$ & $a$ & $\eta$ \\
\hline 17.5 & 0.01981 & 0.450 \\
\hline$q^{2 *}$ & $\eta_{\theta}\left({ }^{\circ} \mathrm{K}\right)$ & $\theta_{1}\left({ }^{\circ} \mathrm{K}\right)$ \\
\hline 0.05 & -660.6 & 989.3 \\
\hline
\end{tabular}

along the streamlines $q^{2}=0.0,0.5$, and 0.9 are shown Figure 3 .

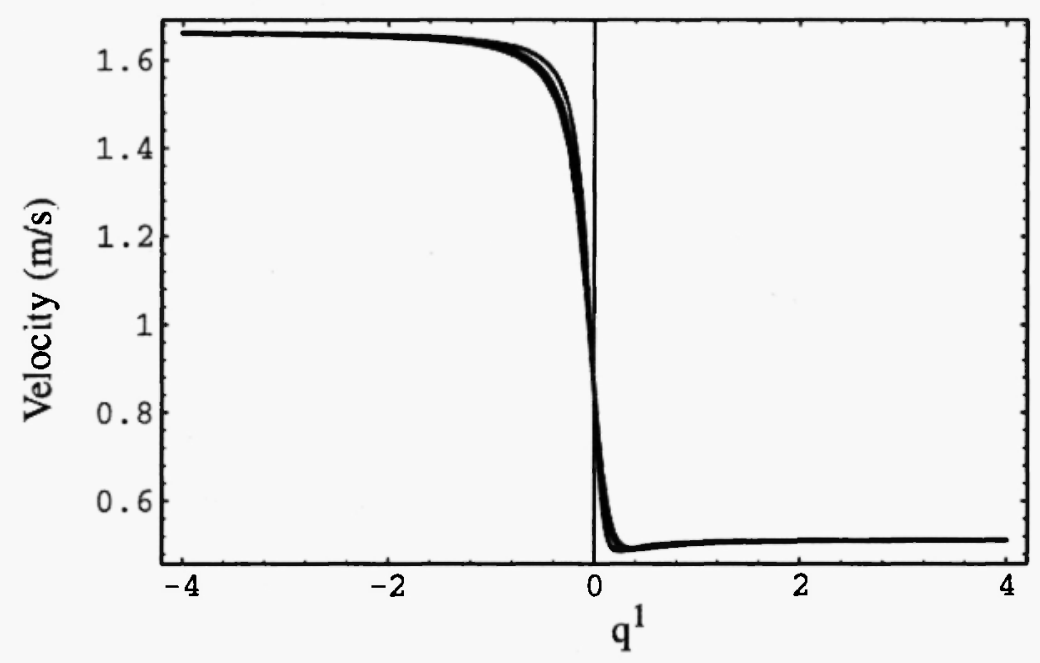

Figure 3 The velocity distributions along typical streamlines in the primary zone

The 2nd invariant of the deformation rate tensor in the primary zone is given in Figure 4. The maximum effective deformation rate is about $2 \cdot 10^{4} s^{-1}$. The deformation rate varies along the directions parallel to the shear plane. The thickness at the outside of the primary zone is greater than that at the inner side of the primary zone. This deformation pattern is in good agreement with the experimental observations in metal cutting. 


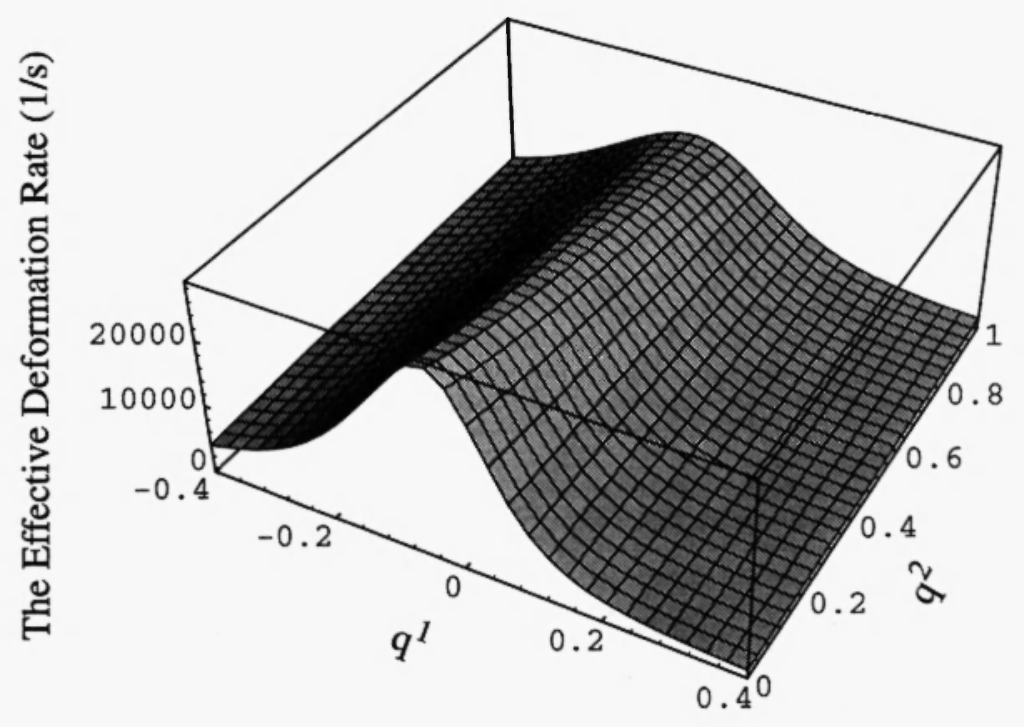

Figure 4 The effective deformation rate in the primary zone

The thickness of the boundary layer adjacent to the chip/tool interface is shown in Figure 5. The average value of the thickness is about one fifth of the undeformed chip thickness. This result agrees well with the experimental results given in [Kim and Marinov, 1995]. The thickness of the boundary layer decreases with the distance away from the tool tip. The velocity along the tool/chip interface is defined by Eq.(18), which is illustrated in Figure 6. The first half of the contact length is almost in a state of seizure. The effective deformation rate in the secondary zone is given in Figure 7.

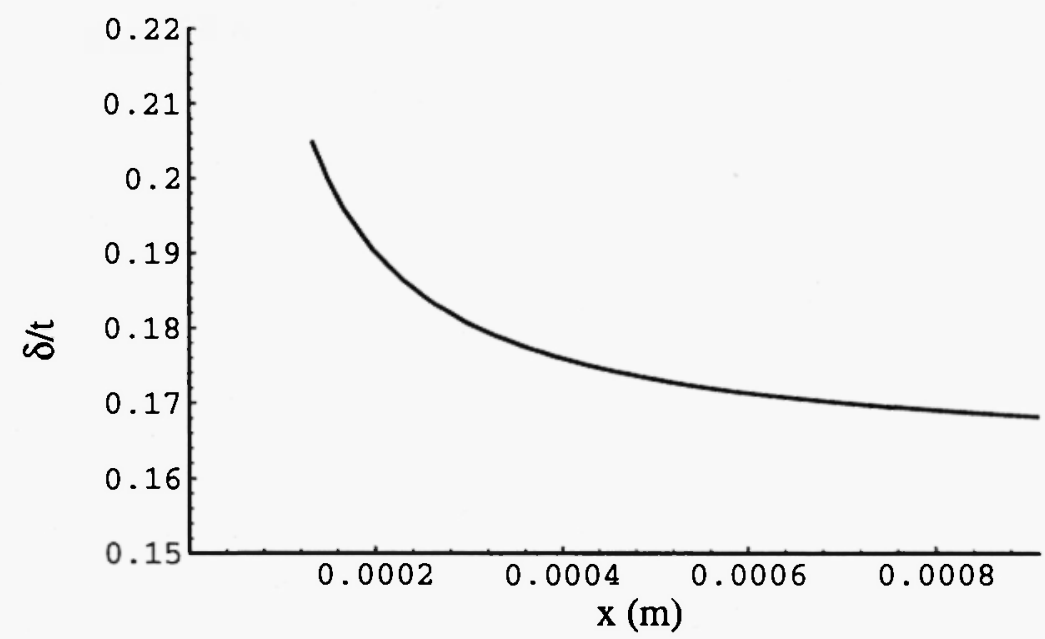

Figure 5 The Thickness of the boundary layer or the secondary zone 


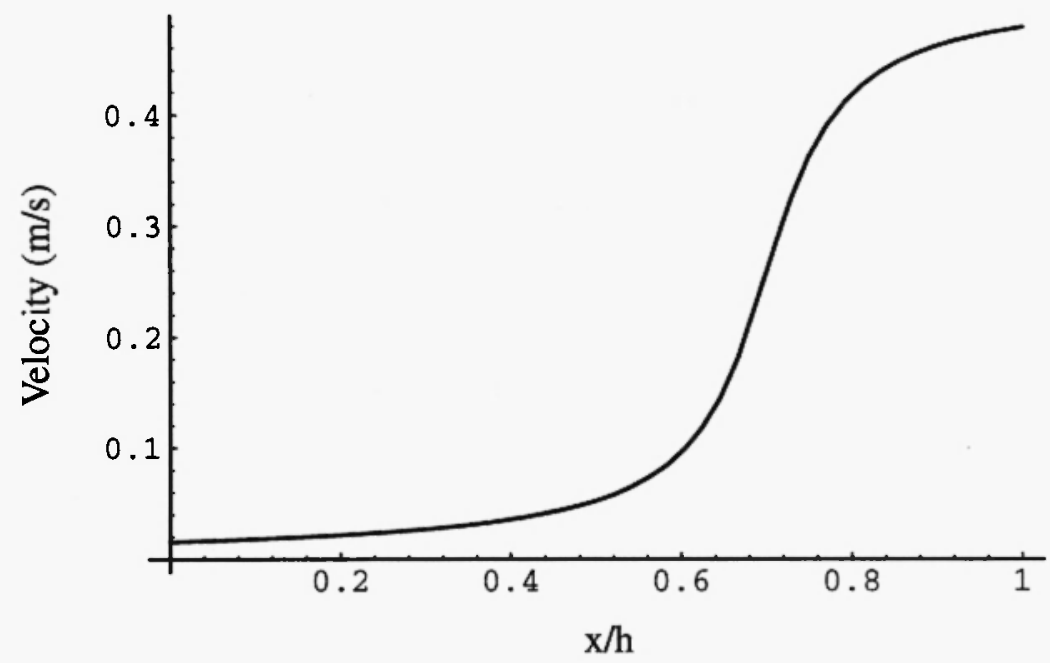

Figure 6 The velocity distribution along the tool/chip interface: $\omega=15.0$ and $b=0.70$

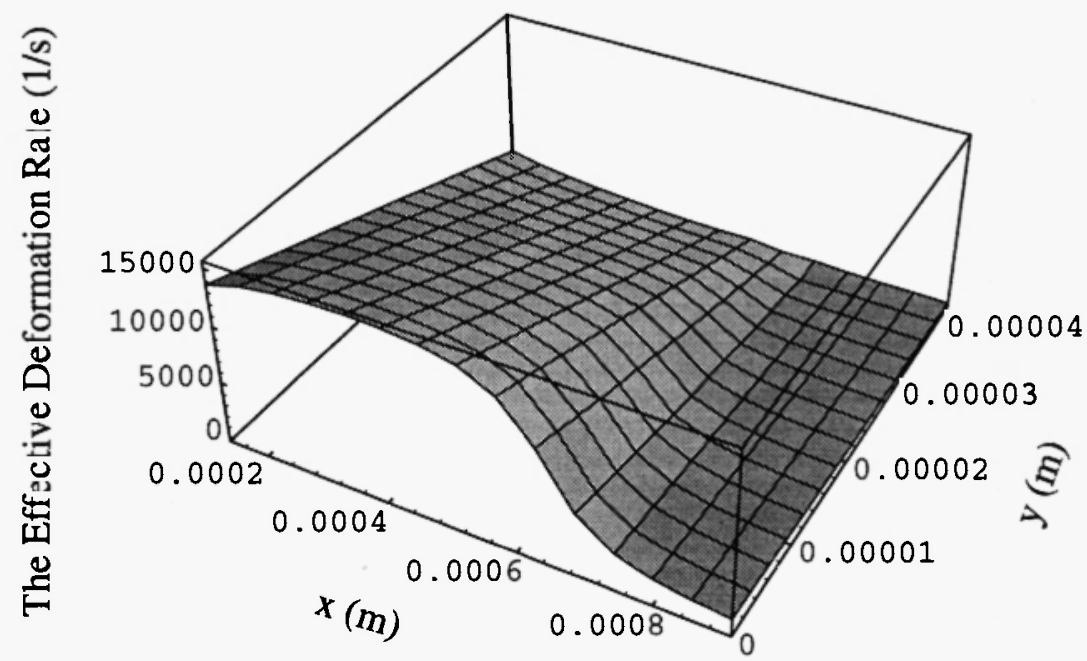

Figure 7 The effective deformation rate in the secondary zone

Due to the assumption of the non-uniform sliding velocity along the contact length, the effective deformation rate is only approximately uniform near the beginning of the tool/chip interface, then goes to zero at the end of the contact length. The maximum value of the effective deformation rate is about $1.5 \cdot 10^{4} \mathrm{~s}^{-1}$. The effective deformation rate at the upper side of the boundary layer is much smaller than that at the tool/chip interface.

The approximate temperature distributions in both the primary zone and the secondary zone are shown in Figures 8 and 9 respectively. At the end of the primary zone, the temperature 
can reach $600^{\circ} \mathrm{C}$, the maximum temperature at the tool/chip interface is about $750^{\circ} \mathrm{C}$, and the final temperature of the formed chip in this case is $720^{\circ} \mathrm{C}$.

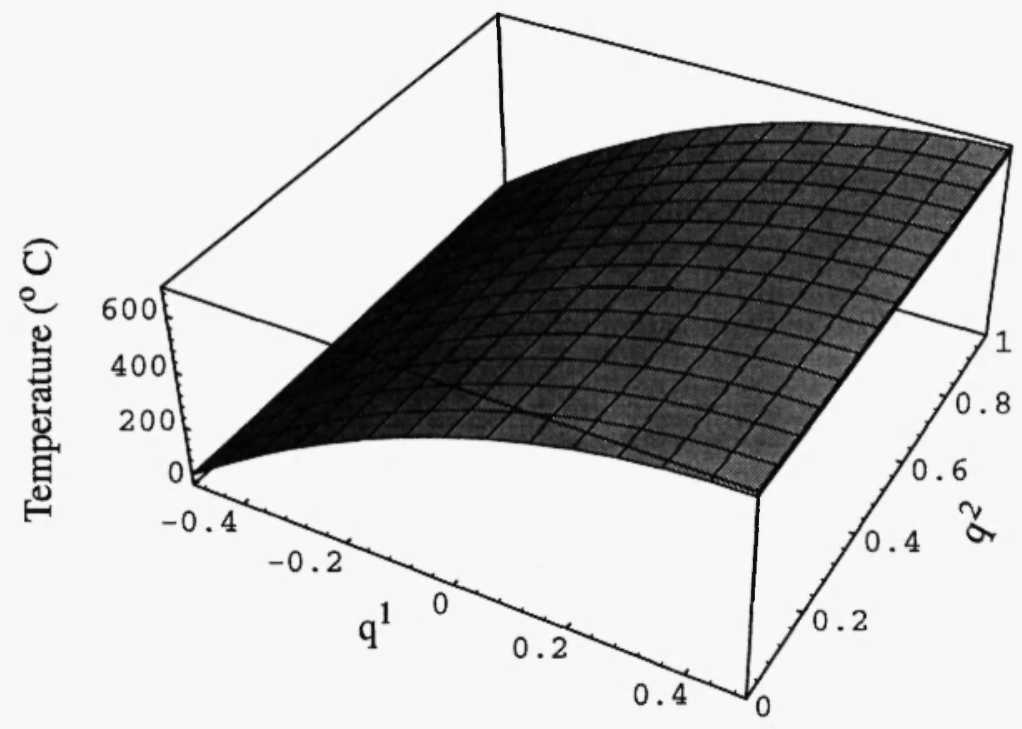

Figure 8 The temperature distribution in the primary zone

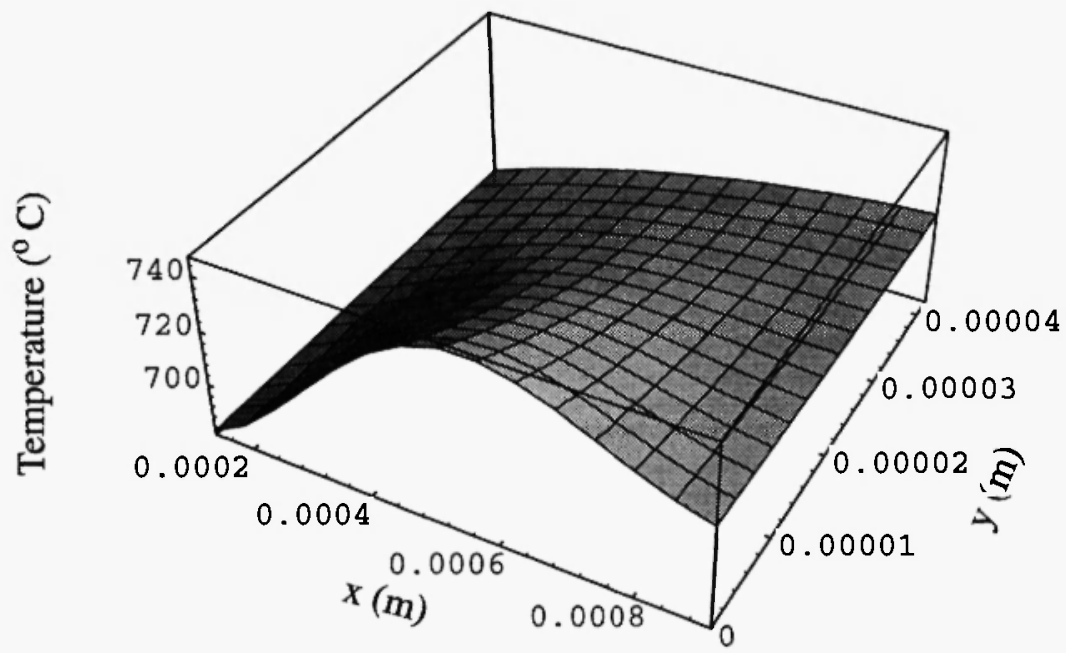

Figure 9 The temperature distribution in the secondary zone

The shear stress distribution along the contact length is given in Figure 10. Due to the varying temperature distribution along the contact length, the shear flow stress is not constant, even for regions with low velocity (close to seizure). And because of sliding, the shear flow stress decreases to zero at the end of contact length. If the sliding velocity is equal to the chip velocity, the shear deformation rate will vanish. From Eq.(21), we know the corresponding shear stress is 
also zero, although in this calculation, the shear stress is not zero due to the sliding velocity is not exactly equal to the chip velocity.

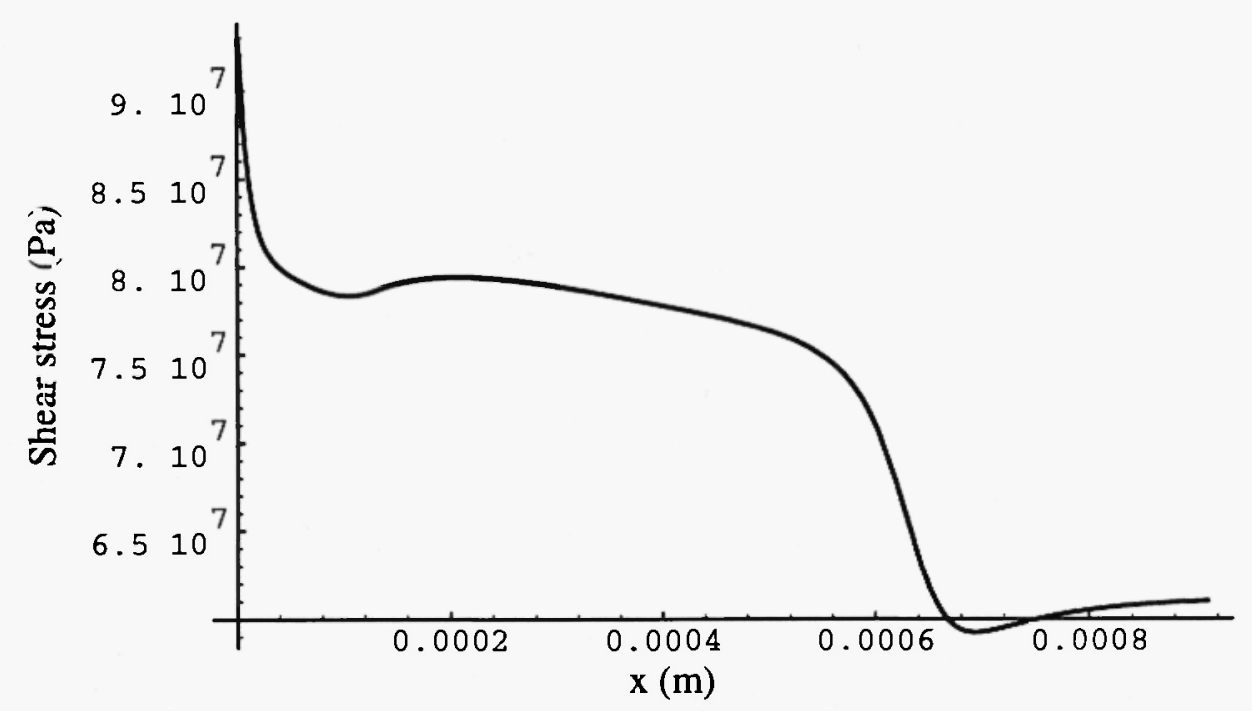

Figure 10 The shear flow stress distribution over the tool/chip interface

For unit width of cut, the work done in the primary zone, secondary zone and at the tool/ chip interface are $4.831 \times 10^{5}, 3.540 \times 10^{4}$ and $1.022 \times 10^{4} \mathrm{Watt} \cdot \mathrm{m}^{-1}$. The work done in the primary zone amounts to $91.0 \%$ of the total work. The work done in the secondary zone and at the tool/chip interface is only about $9.0 \%$ of the total work.

Supposing that the total work done by cutting force, then the specific cutting force is $1268.9 \mathrm{~N} \cdot \mathrm{mm}^{-2}$.

\subsection{Conclusion}

This predictive model underestimates the cutting forces, because that the contact length is much smaller than that indicated in [Kim and Marinov, 1995], and that the constitutive equation used is not suitable, physically the visco-plastic constitutive equation is not rate-sensitive. But if we know the experimental range of the parameters, such as shear angle and contact length, this model can be used to calculate the intensity and shape of the heat sources in machining and better results can be expected. It should be mentioned that the temperature distributions in the primary zone, especially the secondary zone obtained are approximate. Another heat generation model implementing other forms of constitutive equation is currently being developed. Once the heat sources in cutting are determined, the temperature distributions in the tool, chip and workpiece can be calculated by using finite element method.

\subsection{Acknowledgments}


The authors gratefully acknowledge the funding assistance for this research from the NSF-ARPA Machine Tool Agile Manufacturing Research Institute (MT-AMRI) and the NSF Career Award Program (DMI-9502109).

\section{References:}

Boothroyd, G., “Temperatures in Orthogonal Metal Cutting,” Instn. Mech. Engrs.. Vol. 177, No. 29, 1963, p. 789.

Hastings, W. F., P. L. B. Oxley and M. G. Stevenson, "Predicting a Material's Machining Characteristics Using Flow Stress Properties Obtained from High-speed Compression Tests," Proc. Instn. Mech. Engrs. Vol. 188, No.22, 1974, pp. 245-252.

Incropera, F. P., and D. P. DeWitt, Introduction to Heat Transfer, John Wiley \& Sons, 1979.

Jackson, P. S., and P. K. Wright, "Application of Plastic Boundary Layer Theory to Metal Machining," Trans. ASME J. Engng. Ind. Vol.104, 1982, p. 358.

Kim J., and V. R. Marinov, "Material Characterization in the Chip-tool Deformation Zone: An Application of Boundary Layer Theory," Int. J. Mech. Sci. Vol. 37, No. 1, 1995, pp. 91-95.

Lee, B. Y., "Prediction of Specific Cutting Force and Cutting Force Ratio in Cutting," Journal of Materials Processing Technology, Vol. 41, 1994, pp. 71-82.

Lin Z. C., and W. C. Pan, "A Thermalelastic-plastic Large Deformation Model for Orthogonal Cutting with Tool Flank Wear-Part I: Computational Procedures; -Part II: Machining Application,” Int. J. Mech. Sci. Vol. 35, No. 10,1993, pp. 829-850.

Malvern, L. E., Introduction to the Mechanics of a Continuous Medium. Englewood Cliffs, NJ: Prentice-Hall, Inc., 1969.

Marusich, T. D., and M. Ortiz, "A Finite Element Study of Chip Formation in High Speed Machining," Manufacturing_Science and Engineering, ASME. MED-Vol. 2-1/MH-Vol. 31,1995 .

Merchant, M. E., “Mechanics of the Metal Cutting Process,” J. Appl. Phvs.. Vol. 16, 1945, 267318.

Oldroyd, J. G., "Two-dimensional Plastic Flow of a Bingham Solid: A Plastic Boundary Layer Theory for Slow Motion," Proceedings of the Cambridge Philosophical Society. Vol. 43, 1947 , pp. 383-395.

Rapier, A. C., "A Theoretical Investigation of the Temperature Distribution in the Metal Cutting Process," Brit.J. Appl. Phvs. Vol. 5, 1954, p. 400.

Stephenson, D. A., and S. M. Wu, "Computer Models for the Mechanics of Three-dimensional Cutting Processes-Part I: Theory and Numerical Method," Trans. ASME, J. Engng. Ind.. Vol. 110, No. 37, 1988.

Stevenson, M. G., and P. L. B., Oxley, "An Experimental Investigation of the Influence of Speed and Scale on the Strain-Rate in a Zone of Intense Plastic Deformation," Proc. Inst. of Mech. Engrs. Vol. 184, 1979-1980, pp. 561-569. 
Stevenson, M. G., P. K. Wright, and J. G. Chow, "Further Developments in Applying the Finite Element Method to the Calculation of Temperature Distributions in Machining and Comparisons with Experiment," Trans. ASME. J. Engng. Ind.Journal of Engineering for Industry. Vol. 105, 1983, pp. 149-154.

Tay, A. O., Stevenson M. G. and G. de Vahl Davis, "Using the Finite Element Method to Determine Temperature Distributions in Orthogonal Machining," Proc. Inst. of Mech. Engrs.. Vol. 188, 1974, pp.627-638.

Tay, A. O., Stevenson M. G. and G. de Vahl Davis, P. L. B. Oxley, “A Numerical Method for Calculating Temperature Distributions in Machining, from Force and Shear Angle Measurements," International Journal of Machine Tool Design and Research. Vol. 16, 1976, pp. 335-349.

Wolfram, S., Mathematica - A System for Doing Mathematics by Computer. 2nd ed., AddisonWesley Publishing Company, Inc., 1993.

Wright, P. K., "Predicting the Shear Plane Angle in Machining from Work Material Strainhardening Characteristics," Trans. ASME. J. Engng. Ind.. Vol. 104, 1982, p. 285.

\subsection{Nomenclature List}

$$
\begin{aligned}
& \mathrm{x}^{1}, \mathrm{x}^{2}=\text { The coordinates in the reference frame } \\
& \mathrm{q}^{1}, \mathrm{q}^{2}=\text { The coordinates in the curvilinear frame } \\
& \mathrm{t}=\text { The uncut chip thickness } \\
& \alpha=\text { The rake angle } \\
& \phi \quad=\text { The shear angle } \\
& \text { w }=\text { The width of cutting } \\
& \mathrm{h}=\text { The contact length } \\
& \lambda=\text { The mean friction angle over the chip/tool interface } \\
& \mathbf{g}_{\mathrm{i}} \quad:=\text { The covariant base vector in the curvilinear frame } \\
& \tilde{\boldsymbol{g}}=\text { The metric tensor } \\
& \mathrm{g}_{\mathrm{mn}}=\text { The covariant components of the metric tensor } \\
& \mathrm{g}^{\mathrm{mn}}=\text { The contravariant components of the metric tensor } \\
& \mathbf{v} \quad=\text { The velocity vector } \\
& v_{i}=\text { The covariant components of the velocity vector } \\
& v^{i}=\text { The contravariant components of the velocity vector } \\
& v^{<i>}=\text { The physical components of the velocity vector } \\
& V_{o}, V_{c}=\text { The cutting speed and the final velocity of the chip } \\
& \rho_{o}, \rho_{c}=\text { The initial and final density of the chip } \\
& \tilde{D} \quad=\quad \text { The deformation rate tensor in the primary zone } \\
& \mathrm{D}_{\mathrm{ij}}=\text { The covariant components of the tensor } \tilde{D} \\
& \mathrm{D}^{\mathrm{ij}}=\text { The contravariant components of the tensor } \tilde{D}
\end{aligned}
$$


$\Pi_{\mathrm{D}} \quad=\quad$ The second invariant of the tensor $\tilde{D}$

$\tilde{T}=$ The deviatric Cauchy stress tensor

$\mu_{0}, \tau_{0}=$ The material related functions respect to temperature

$\mathrm{C}_{\mathrm{p}}=$ The specific heat capacity

$\mathrm{k}=$ The thermal conductivity

$\delta=$ The thickness of the boundary layer

$\mathrm{q}^{1^{*}} \quad=$ A parameter to determine the initial position of the boundary layer

$\mathrm{q}^{2^{*}}=$ A parameter to determine the thickness of the boundary layer

$v_{x}, v_{y}=$ The components of velocity vector in the boundary layer

$\mathrm{V}_{\mathrm{w}}=$ The velocity distribution along the chip/tool interface

$\tilde{d} \quad=$ The deformation rate tensor in the boundary layer

$\mathrm{d}_{\mathrm{ij}} \quad=$ The components of the tensor $\tilde{d}$

$\Pi_{\mathrm{d}} \quad=\quad$ The second invariant of the tensor $\tilde{d}$

$\tau_{x y}=$ The shear stress over the chip/tool interface

$\theta=$ The temperature

$\eta_{\theta}=$ A parameter to determine the temperature distribution in the primary zone

$\theta_{1}=$ A parameter to determine the temperature distribution in the secondary zone

$\mathrm{a}, \eta, \mathrm{C}=$ The parameters to define the streamline

$\psi_{\mathrm{p}} \quad=$ The dissipated work per unit volume in the primary zone

$\psi_{s}=$ The dissipated work per unit volume in the secondary zone

$\psi_{\mathrm{f}}=$ The friction work per unit area generated over the chip/tool interface

$\mathrm{W} \quad=$ The overall work per unit width of cutting done in cutting

$\mathrm{W}_{\mathrm{s}} \quad=$ The dissipated work in the secondary zone

$\mathrm{W}_{\mathrm{f}}=$ The friction work done over the chip/tool interface

$\mathrm{J}=$ The Jacobian determinant between $\left(\mathrm{x}^{1}, \mathrm{x}^{2}\right)$ and $\left(\mathrm{q}^{1}, \mathrm{q}^{2}\right)$ 
